\title{
Empleo de paneles compuestos por subproduc- tos de centrales térmicas en fachadas trasdosadas
}

\section{Facade solutions using panels made of power plant byproducts}

\author{
M. D. Alba $^{(*)}, \underline{\text { M. Marrero }}^{(*)}$, C. Leiva ${ }^{(* *)}$, M. V. Montes ${ }^{(*)}$, L. Vilches $^{(* *)}$
}

RESUMEN

Se presentan dos nuevas tipologías de paneles para trasdosado de fachadas que proporcionan como mejora ambiental la combinación de materiales tradicionales con nuevos materiales reciclados obtenidos de residuos procedentes de la combustión de orujillo y de carbón en centrales térmicas. Con anterioridad a su uso, dichos residuos han sido sometidos a exhaustivos ensayos químicos y los paneles fabricados a partir de los mismos a ensayos mecánicos, térmicos y de resistencia al fuego que justifican su empleo. Posteriormente, los nuevos paneles se han implementado como trasdosado de una solución de fachada convencional para verificar su viabilidad funcional y el buen comportamiento aislante del conjunto. De los resultados obtenidos se extrae que los paneles fabricados con cenizas de orujillo tienen un mejor comportamiento mecánico y térmico que los realizados con cenizas de carbón, proporcionando una alternativa constructiva más sostenible a través de reducir el consumo de materias primas y eliminar residuos industriales.

113-114

Palabras clave: Fachadas; aislamiento térmico; materiales reciclados; cenizas de orujillo; cenizas de carbón.

\section{SUMMARY}

Two new recycled gypsum boards are proposed which have a better environmental performance due to the fact that they are made of traditional materials and new recycled ones derived from biomass (waste generated during the extraction of olive pomace) and the coal combustion produced in power plants. Before using these wastes as raw materials, they have been submitted to chemical analysis and the panels made of them have been tested to prove their mechanical, thermal and fire performance. Subsequently, the new recycled panels have been implemented in a traditional facade solution to verify their functional and thermal feasibility. The results obtained show that the panels made of biomass ashes have a better mechanical and thermal performance than those made of coal ashes and that they can provide a sustainable alternative to the traditional facade materials due to the reduction of raw materials consumption and the elimination of industrial waste.
Keywords: Facades; thermal insulation; recycled materials; biomass ashes; coal ashes. 


\section{INTRODUCCIÓN}

La importancia estética y funcional de la fachada la han convertido en un elemento arquitectónico sujeto a una incesante y notable evolución a lo largo de los siglos; su condición de soporte para expresar la sucesión de estilos arquitectónicos de distintas épocas ha ocasionado una continua transformación de la fachada íntimamente ligada a la evolución experimentada por las técnicas de construcción de este elemento arquitectónico (1).

Desde las fachadas macizas, se ha trabajado en la evolución técnica de éstas con el fin mejorar el confort en el interior de los edificios (2). Una primera variante de estas fachadas macizas la desarrollaron los griegos con la creación del muro de dos hojas, formado por dos paramentos trabados entre los que se insertaban bastos mampuestos y mortero que se encargaban de asumir la misión portante del cerramiento. Pero fueron los romanos quienes, contando con las ventajas del descubrimiento del cemento hidráulico, material con alta capacidad impermeabilizante y resistencia, fueron capaces de dotar a esta tipología de cerramiento de estanqueidad y un cierto nivel de aislamiento acústico (3).

No fue hasta el siglo XIX, cuando, rompiendo con la cultura arquitectónica arraigada hasta el momento, se demuestra que la fachada hueca presenta mejores propiedades que la fachada maciza, mostrando una notable mejora en cuanto a control de humedades y aislamiento térmico. En el caso particular de España, tras la guerra civil, cobra fuerza la presencia del cerramiento de doble hoja y a partir de 1940 la reglamentación normativa aplicada a la protección pública de las viviendas contempla el análisis del comportamiento higrotérmico de los cerramientos como niveles mínimos exigibles de calidad (4). En la fachada de doble hoja también aparece el empleo de paneles de yeso laminado, un tablero de dos cartones con alma de yeso, inventado en Estados Unidos con el fin de sustituir a los tableros de madera y aumentar la resistencia al fuego en caso de incendio y que en países mediterráneos, de tradición constructiva en albañilería, produce un efecto importante tendente a la industrialización del sistema constructivo (5).

Pese al gran avance que el sistema de doble hoja supuso para el desarrollo de la técnica, en cuanto a construcción de fachadas, seguían surgiendo problemas de funcionalidad: se generaban condensaciones, movimientos diferenciales y puentes térmicos. Con la aparición de los materiales aislantes, tales como el corcho y posteriormente toda la gama de poliuretanos, poliestirenos y polietilenos y su colocación en el interior del cerramiento, consiguen solucionarse algunas de las problemáticas descritas, no obstante siguen existiendo patologías intrínsecas del sistema constructivo, que hacen indudable la necesidad de la continuidad evolutiva de los sistemas de fachadas de modo que permitan salvar sus debilidades hasta conseguir la solución óptima (3).

Con la llegada del Código Técnico de Edificación (CTE) (6), se consiguen establecer las bases para las soluciones constructivas viables actualmente, en este caso de la envolvente del edificio, para lo cual deben cumplirse las exigencias básicas en cuanto a protección frente al ruido, salubridad y ahorro de energía, funciones todas ellas íntimamente ligadas a las fachadas. Es el proceso de diseño de una fachada, el que afecta en gran medida al futuro ahorro energético del edificio en su periodo de utilización. La exigencia básica de Ahorro de Energía HE1: Limitación de la demanda energética del CTE, establece la necesidad de disponer en los edificios de una envolvente de características tales que limite adecuadamente la demanda energética esencial para alcanzar el necesario confort térmico interior, mediante un apropiado grado de aislamiento, permeabilidad al aire y exposición a la radiación solar.

El compromiso ambiental actual que debe tener la edificación, y en el caso particular de las fachadas, no sólo incluye el ahorro de energía en el uso del edificio sino también se deben reducir los impactos ambientales asociados a su construcción. En esta línea el empleo de materiales reciclados en los nuevos productos de construcción constituye una forma de construir más sostenible y respetuosa con el entorno, al conseguir simultáneamente reducir dos grandes impactos medioambientales derivados de la actividad humana, el ingente consumo de recursos naturales y la masiva generación de residuos. Así, en la actualidad, en el sector de la edificación se percibe la incipiente proliferación de nuevos productos de construcción que incorporan materiales reciclados (pétreos, plásticos, madera, vidrio, papel, etc.), gestándose lo que se ha venido en denominar una nueva generación de Eco-productos que aúnan eficiencia y compromiso medioambiental (7).

En este sentido, cabe destacar el uso de cenizas de carbón en hormigones (8) y como materia prima para la fabricación de cementos desde hace muchos años (9). No obstante, el creciente aumento de cenizas generadas, hasta más de 64 millones de 
toneladas en Europa en 2005 (10), debido al incremento de la demanda energética mundial, y la aparición de nuevos tipos de cenizas procedentes de combustibles más ecológicos, tales como la biomasa, no aptas para ser empleadas en cementos y hormigones, hacen insuficiente la cantidad de cenizas recicladas, por lo que es necesario buscar nuevas aplicaciones donde reutilizar este tipo de subproductos, tales como estabilización de subsuelos, fabricación de materiales cerámicos, materiales resistentes al fuego o actividades agrícolas (10).

En el presente trabajo se propone el reciclado de este tipo de residuo en paneles de yeso para trasdosados. Para este estudio se ha escogido una ceniza típica de combustión de carbón así como ceniza procedente de la combustión de orujillo, un residuo procedente de la extracción de aceite de oliva, por ser la biomasa más abundante presente en la región donde se ha desarroIlado este estudio, ya que las cenizas no deben ser transportadas a zonas muy lejanas de su zona de producción.

Se parte de estudios previos llevados a cabo por parte de los autores de este trabajo donde se han analizado las posibilidades de fabricar paneles compuestos mayoritariamente por cenizas procedentes de distintos procesos térmicos $(11,12,13,14)$. Si bien la adición de residuos industriales a materiales de construcción tales como los hormigones y morteros es objeto de numerosas investigaciones $(15,16,17)$, en el campo de la incorporación de residuos industriales o de cualquier otra naturaleza a paneles de yeso para trasdosados aún queda mucho por experimentar. Entre los principales antecedentes a este trabajo encontramos las investigaciones de González Madariaga y Lloveras Macia (18).

Finalmente, los nuevos materiales reciclados se emplean en soluciones constructivas de fachada, a través de prototipos, que cumplen con el Código Técnico de la Edificación en la región evaluada y proporcionan un valor añadido a la envolvente de los edificios mediante la reducción del consumo de materias primas y la eliminación de residuos industriales.

\section{CARACTERIZACIÓN DE LOS MATERIALES RECICLADOS PROCEDENTES DE CENIZAS DE ORUJILLOY DE CARBÓN}

\subsection{Composición de los paneles con materiales reciclados}

Los dos materiales objeto de aplicación en el presente trabajo han sido desarrollados en forma de paneles de $60 \times 60 \mathrm{~cm} \mathrm{y}$
1,50 cm de espesor, en cuya composición sólida general encontramos cenizas, conglomerante y aditivos.

Los objetivos perseguidos con el desarrollo de estos nuevos productos son la creación de nuevos materiales de construcción elaborados a partir de pastas compuestos por un alto porcentaje de residuos (> 50\%p (porcentaje en peso)), que presenten una baja densidad $\left(<900 \mathrm{~kg} / \mathrm{m}^{3}\right)$, una alta resistencia al fuego (conferido por la alta proporción de cenizas en su composición), una resistencia a compresión superior a 1 MPa y, por último, un sencillo método de fabricación, siguiendo la línea de trabajo de del Río Merino et al. (19, 20), García Santos (21) y la tesis doctoral de Leiva (11).

En primer lugar se han seleccionado las cenizas; de entre las cenizas analizadas, se ha optado por la ceniza volante de carbón de la central Térmica de Los Barrios (Cádiz, Andalucía) (11) y la ceniza de orujillo de la Central Térmica de Las Lomas (Jaén, Andalucía) que se analiza por primera vez como material de construcción.

Para alcanzar los objetivos planteados las pastas se han fabricado con un $60 \%$ p de cenizas, con vermiculita sin moler como aditivo y yeso como material conglomerante, por aportar mayor capacidad aislante manteniendo unas propiedades mecánicas aceptables. Las características del yeso empleado en la fabricación de las pasta es un yeso comercial de acuerdo con la norma UNE 102001 (22) y la vermiculita corresponde a vermiculita exfoliada comercial (VERLITE S.A.) de forma que el $84,9 \%$ p presenta un tamaño de partícula menor de 1,41 mm. Para satisfacer que los materiales cumplieran una resistencia a compresión superior a $1 \mathrm{MPa}$ se han reforzado con fibras, una parte en forma de fibra monofilamento de polipropileno que mejora su resistencia a compresión y otra parte en forma de malla de fibra de vidrio que incrementa la resistencia a flexión e impacto. Un estudio previo (11) sobre la influencia del tipo de fibras y su dosificación en este tipo de pastas ha sido Ilevado a cabo para su correcta selección, de forma que se alcancen unas óptimas propiedades mecánicas.

Tras un estudio de las diferentes posibilidades (11), en función de las premisas anteriormente comentadas, se ha optado por elegir las composiciones mostradas en la Tabla 1, de forma que ambas presentan los mismos componentes, diferenciándose sólo en el tipo de ceniza (P1 = panel con ceniza de orujillo y P2 = panel con ceniza de carbón), de forma que será posible eva- 
luar la influencia de éstas. Para la fabricación de los paneles, se han pesado los materiales en las dosificaciones mostradas en la Tabla 1 y se han mezclado hasta obtener un material homogéneo. Posteriormente se han mezclado con agua (relación agua/sólidos en P1 $=0,45$ y en P2 $=0,42$ ) hasta obtener una pasta homogénea, la cual ha sido introducida en los diversos moldes necesarios para fabricar las muestras para las distintos ensayos.

Tabla 1

Composición de las pastas que forman los paneles

\begin{tabular}{|l|r|r|}
\hline \multicolumn{1}{|c|}{ Componentes sólidos (\% p) } & \multicolumn{1}{c|}{ P1 } & \multicolumn{1}{c|}{ P2 } \\
\hline Cenizas de orujillo & 60,0 & 0,0 \\
\hline Cenizas de carbón & 0,0 & 60,0 \\
\hline Yeso & 30,0 & 30,0 \\
\hline Vermiculita sin moler & 9,5 & 9,5 \\
\hline Fibra de vidrio monofilamento/ mallado & 0,5 & 0,5 \\
\hline
\end{tabular}

\subsection{Características técnicas de los paneles}

\subsubsection{Propiedades físicas y mecánicas}

En la Tabla 2 se muestran la densidad, capacidad de absorción de agua y las principales propiedades mecánicas de los paneles desarrollados, de forma que se comparan los valores obtenidos con los de la normativa correspondiente a paneles de yeso, el material más similar al desarrollado en este estudio (23).

Tabla 2

Análisis comparativo de los paneles de cenizas de orujillo (P1) y los paneles de cenizas de carbón (P2)

\begin{tabular}{|l|c|c|}
\hline \multicolumn{1}{|c|}{ Propiedades } & P1 & P2 \\
\hline Densidad $\left(\mathrm{kg} / \mathrm{m}^{3}\right)$ & 800 & 850 \\
\hline Capacidad de absorción de agua (\%) & 32,6 & 28,0 \\
\hline Resistencia a compresión (MPa) & 1,2 & 0,8 \\
\hline Resistencia a flexión $(\mathrm{MPa})$ & 1,8 & 0,9 \\
\hline Dureza superficial (Shore C) & 39 & 32 \\
\hline Resistencia al impacto $(\mathrm{cm})$ & 2,4 & 2,2 \\
\hline Conductividad térmica $(\mathrm{W} / \mathrm{mK})$ & 0,25 & 0,33 \\
\hline
\end{tabular}

Desde el punto de vista de la densidad, los paneles P1 pueden ser catalogadas como de baja densidad $\left(\leq 800 \mathrm{~kg} / \mathrm{m}^{3}\right)$, mientras que las P2 pueden ser catalogadas como de densidad media (entre 800 y 1100 kg/ $\left.\mathrm{m}^{3}\right)(23)$.

La capacidad de absorción de agua se ha determinado de acuerdo a la norma UNEEN 12859:2009 (23). La alta capacidad de absorción de agua que presentan ambos paneles es debida, probablemente, a su baja densidad. La alta porosidad que confiere a estos materiales la adición de vermiculita limita su empleo como material no hidrófugo al rebasar el 5\% de absorción de agua exigido por la normativa (23).

Los ensayos de resistencia a compresión se han realizado según lo establecido por la norma ASTM E761 - 92(2005) (24), mediante una máquina de ensayo (Suzpecar, MEM-102 / 50t) con probetas cilíndricas de $40 \mathrm{~mm}$ de alto y $35 \mathrm{~mm}$ de diámetro. El resultado de la resistencia a compresión de los paneles de cenizas de orujillo ha sido superior a la de las cenizas de carbón; la contribución de las fibras monofilamento de polipropileno ha tenido un efecto muy positivo en la resistencia a compresión de forma que casi ha triplicado estos valores respecto a la misma composición sin fibra en ambos casos (9).

Los ensayos de la resistencia a flexión se han realizado según lo establecido en la norma ASTM C348 - 08 (25), mediante la misma máquina utilizada en los ensayos de resistencia a compresión, pero en esta ocasión con probetas de $4 \times 4 \mathrm{~cm}$ de base y 14 $\mathrm{cm}$ de alto. Los resultados de la resistencia a flexión de los paneles presentan un valor superior en el caso del panel con ceniza de orujillo y, en ambos casos, se sitúan por encima de los 0,6 MPa que impone la norma (23) para productos de densidad media, ya que para productos de yeso de baja densidad no se especifican valores.

Debido al propósito de emplear los paneles en el presente trabajo como material de construcción, y dado que éstos podrían ser objeto de impacto, se hace necesario el análisis de la dureza superficial del material, según lo establecido en la normativa UNE-EN 12859:2009 (23), donde se especifica el método de resistencia a la penetración dado por el panel. La dureza superficial de los paneles con cenizas de biomasa presenta nuevamente un valor ligeramente superior al de las cenizas de carbón, si bien los valores obtenidos son ligeramente inferiores al límite de 40 Shore $\mathrm{C}$ establecido en la norma (23) para materiales de baja densidad. Este resultado muestra que el producto desarrollado puede soportar impactos superficiales (puntillas, taladro, etc.) sin presentar grandes riesgos de rotura.

La resistencia al impacto se ha medido de acuerdo con la normativa UNE-EN 12859:2009 (23), consistente en la medición de la impresión que aparece en el panel cuando éste se ve sometido al impacto de una bola de acero con un potencial de energía de $245 \mathrm{~J}$. El resultado de la prueba de resistencia al impacto en los paneles con cenizas de biomasa es ligeramente superior al de las cenizas de carbón, siendo los valores obtenidos a su vez superiores al de otros productos comerciales (2,0 y 2,2 cm).

La conductividad térmica ha sido determinada acorde a la UNE-EN 993-15:2005, mediante la técnica de hilo caliente parale- 
lo (26). La norma UNE-EN 12859:2009 (23) especifica que para productos de baja densidad $\left(<800 \mathrm{~kg} / \mathrm{m}^{3}\right)$ la conductividad térmica debe ser inferior a $0,26 \mathrm{~W} / \mathrm{mK}$, lo cual es satisfecho para $\mathrm{P} 1$, mientras que para productos de densidad media (entre 800 y $1000 \mathrm{~kg} / \mathrm{m}^{3}$ ) deben de presentar valores inferiores a 0,39 W/mK, lo cual es también satisfecho por P2.

\subsubsection{Resistencia al fuego}

El ensayo de resistencia el fuego de los paneles ha sido realizado según la UNE-EN 1363-1:2000 (27) en un horno eléctrico capaz de simular un ensayo de resistencia al fuego, de forma que el equipo permite registrar los datos tanto de la cara expuesta a la curva normalizada del fuego como de la que no lo está, mediante termopares $\mathrm{S}$ y Pt-100 $(9,28)$. Los ensayos han sido llevados a cabo en paneles de $28 \mathrm{~cm}$ de alto por $18 \mathrm{~cm}$ de ancho y $2 \mathrm{~cm}$ de espesor. Con el objetivo de comparar los resultados obtenidos en estas paneles con el de otros materiales ya empleados comercialmente, en la Figura 1 se muestran los resultados de otros dos materiales, uno compuesto de silicato cálcico (Comercial 1) y otro compuesto por yeso y vermiculita (Comercial 2) (28); tal y como se puede observar en la Figura 1, los materiales presentados en este trabajo presentan una resistencia al fuego del mismo orden que los ya empleados comercialmente. otros productos comerciales con similar aplicación (11).

Durante la realización del ensayo no se produjeron emisiones de gases nocivos, manteniendo los paneles la estabilidad mecánica, antes, durante y después del ensayo, tanto en la cara expuesta como en la no expuesta, sin que se apreciase pandeo en las mismas.

\subsubsection{Lixiviación}

Con el fin de establecer un proceso de reutilización de residuos procedentes de la combustión de biomasas en construcción, al no existir una legislación específica en España que establezca que ensayos y límites deben cumplir los materiales de construcción que incorporan residuos, se ha optado por seguir los pasos establecidos en la legislación holandesa. El reciclado de materiales secundarios en el sector de la construcción es muy común en Holanda. Más del $10 \%$ de todos los materiales granulares usados en construcción en dicho país son materiales reciclados. El Soil Quality Decree (29) contiene las reglas y procedimientos relacionados con el uso de dichos materiales con el objetivo de prevenir la contaminación del suelo y de las aguas superficiales. El decreto obliga a la realización de un ensayo de lixiviación normalizado, tipo tanque, para materiales reciclados, el ensayo NEN 7345 (30).

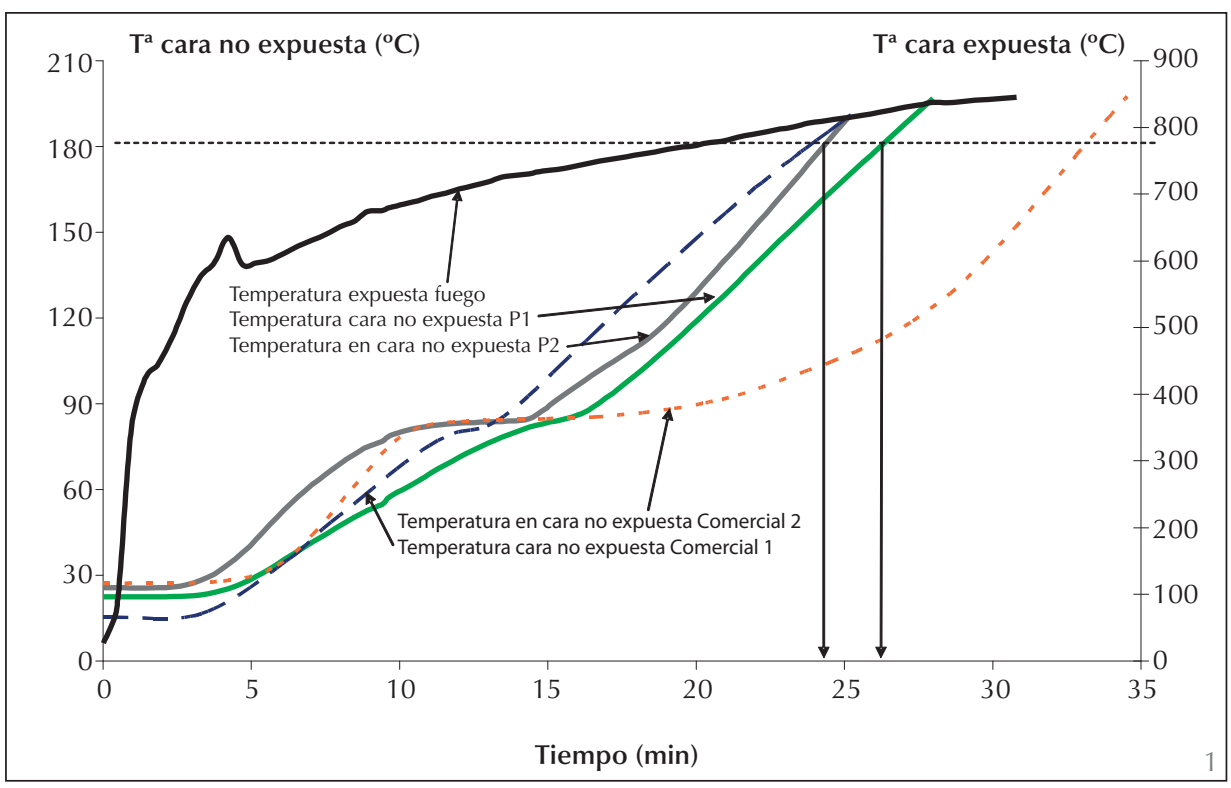

De los resultados obtenidos se extrae que el tiempo necesario para alcanzar los $180{ }^{\circ} \mathrm{C}$ en la cara no expuesta a la curva normalizada del fuego es superior en los paneles de cenizas de orujillo al de las de las cenizas de carbón, siendo la resistencia al fuego obtenida en estos paneles similar e incluso superior a la de
Dado que las cenizas estudiadas son sólo uno de los componentes de los productos P1 y P2 (aunque sea el mayoritario), se ha considerado razonable el prestar una mayor atención a la lixiviación del producto conformado frente a la de las propias cenizas. Así, en la Tabla 3 se muestran los resultados de las concentraciones de metales 
2. Cerramiento de doble hoja con cámara de aire no ventilada, con su hoja principal e interior de fábrica de ladrillo.

3. Cerramiento de doble hoja con cámara de aire no ventilada, con su hoja principal de fábrica de ladrillo y su hoja interior de yeso laminado.

4. Cerramiento de doble hoja sin cámara de aire, con su hoja principal e interior de fábrica de ladrillo. acumulados según se indica en el ensayo NEN 7345 de los paneles, cuyo objetivo es simular el lixiviado de componentes inorgánicos en materiales conformados y monolíticos en función del tiempo. El ensayo se realiza en un periodo de 64 días, manteniendo las probetas completamente sumergidas en fluido lixiviante (agua acidificada $\mathrm{pH}=4$ ) y reponiendo el fluido cada cierto tiempo. Posteriormente, se miden las concentraciones de los componentes lixiviados en las distintas fracciones del eluato (lixiviado). El valor del $\mathrm{pH}$ al que tiene lugar la lixiviación viene determinado por el propio material. De los datos incluidos en la Tabla 3 se puede concluir que los productos analizados no presentan inconveniente para su aplicación en construcción, por lo que respecta a los metales, según la normativa holandesa (29), ya que el impacto ambiental de ambos es mínimo, al no superarse en ningún caso los límites del decreto y encontrarse, además, una gran mayoría de los metales analizados por debajo del límite de detección de la técnica analítica empleada.

\section{DESARROLLO DE LAS SOLUCIONES DE FACHADAS TRASDOSADAS}

3.1. Identificación de los sistemas constructivos de fachadas tipo en Sevilla y caracterización de su grado de aislamiento

Es sabido que el equilibrio energético de un edificio, depende en buena medida del aislamiento térmico que su fachada o cerramiento exterior proporcione. Este aislamiento térmico viene cuantificado por lo que se conoce como transmitancia térmica $(U)$ de los cerramientos, parámetro que mide el flujo de calor, en régimen estacionario, dividido por el área y la diferencia de temperaturas de los medios situados a cada lado de la envolvente del edificio y cuya unidad de medida es $\mathrm{W} / \mathrm{m}^{2} \mathrm{~K}(6)$.

El primer paso del presente estudio es la caracterización de las tres soluciones constructivas de fachadas (figuras 2, 3 y 4) más empleadas en Sevilla identificadas en los trabajos de Mercader et al. en los que se evalúan datos de la Gerencia Municipal de Urbanismo de Sevilla(31, 32).

Tabla 3

Resultado y límites de los metales para los lixiviados obtenidos en el ensayo NEN 7345

\begin{tabular}{|c|c|c|c|c|c|c|c|c|c|c|c|c|c|c|c|}
\hline Componente & Sn & As & $\mathrm{Hg}$ & Se & Mo & $\mathrm{Sb}$ & Zn & $\mathbf{P b}$ & Cd & Co & $\mathrm{Ni}$ & V & $\mathrm{Cu}$ & Ba & $\mathrm{Cr}$ \\
\hline Acumulado P1 (mg/m²) & $<0,1$ & 9,6 & $<0,05$ & $<1$ & $<2$ & $<0,2$ & 29 & 9,6 & 1,0 & $<0,1$ & 1,9 & 2,5 & 51 & 17 & 45 \\
\hline Acumulado P2 $\left(\mathrm{mg} / \mathrm{m}^{2}\right)$ & $<0,11$ & $<6,5$ & $<0,05$ & 1,21 & 2,5 & 0,39 & 11,7 & 3,8 & $<0,1$ & $<0,1$ & $<0,52$ & 3,4 & $<0,3$ & $<1,7$ & 12,5 \\
\hline Límites SQD $\left(\mathrm{mg} / \mathrm{m}^{2}\right)$ & 50 & 260 & 1,4 & 4,8 & 144 & 8,7 & 800 & 400 & 3,8 & 60 & 81 & 320 & 98 & 1500 & 120 \\
\hline
\end{tabular}
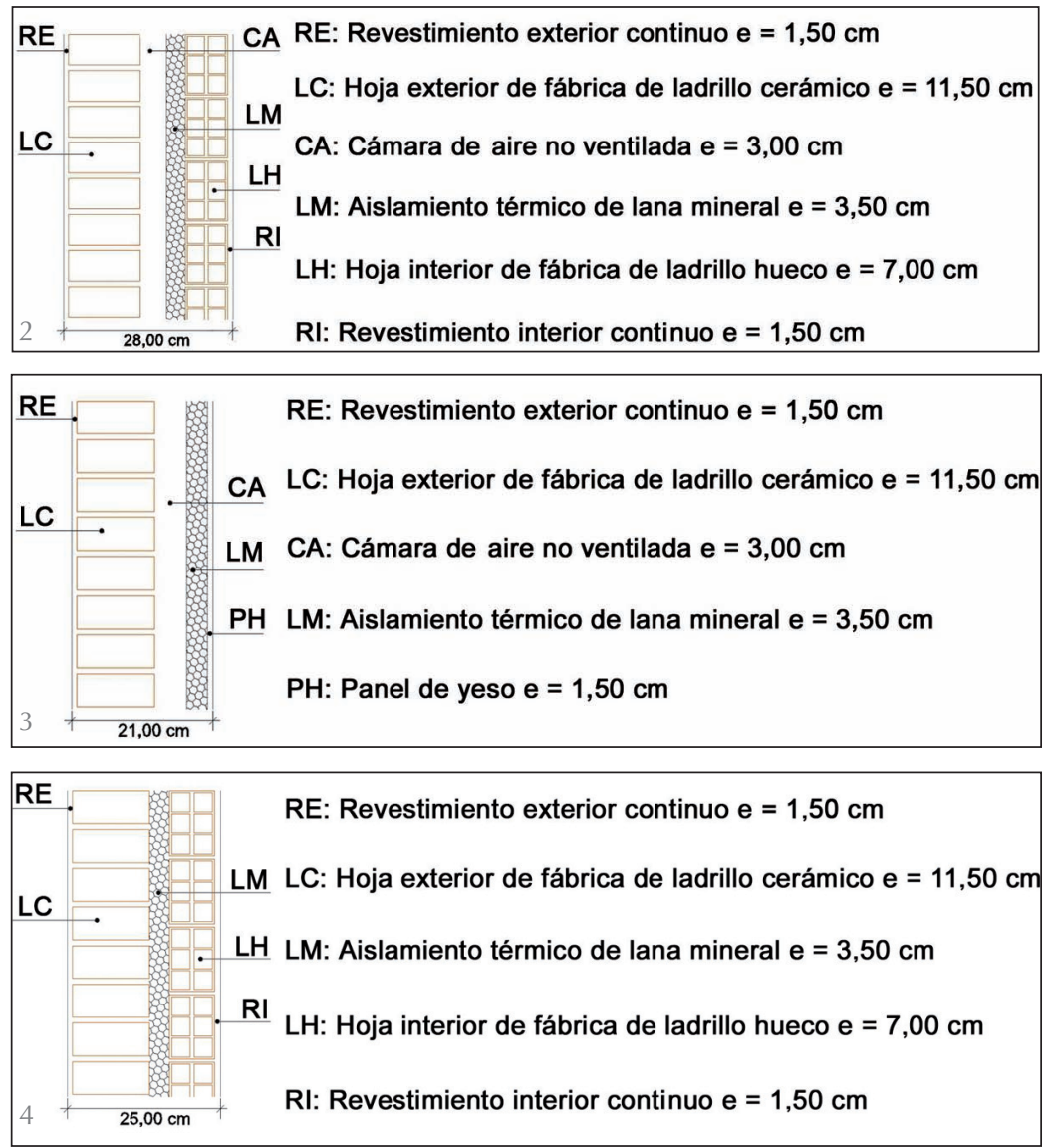

Para llevar a cabo la evaluación se ha empleado como guía el Catálogo de Elementos Constructivos del Código Técnico de Edificación (CTE) (33). De estas tres soluciones se han obtenido sus transmitancias térmicas, con el fin de conocer el grado de aislamiento térmico de estos cerramientos, y se ha comprobado que se encuentran por debajo del valor límite fijado por el CTE para la zona climática de Sevilla, la zona B4, en la cual la transmitancia térmica de muros de fachada presenta una limitación de UMlim $=0,82 \mathrm{~W} / \mathrm{m}^{2} \mathrm{~K}$.

El proceso de cálculo de las trasmitancias térmicas de la parte opaca de los cerramientos en contacto con el aire exterior se encuentra detallado en el Documento Básico HE. Ahorro de energía (6) a partir de la definición de los siguientes parámetros:

- Transmitancia térmica

$(\mathrm{U}): \mathrm{U}\left(\mathrm{W} / \mathrm{m}^{2} \mathrm{~K}\right)=1 / \mathrm{RT}$

- Resistencia térmica total de un componente construido por capas homogéneas (RT): $\mathrm{RT}\left(\mathrm{m}^{2} \mathrm{~K} / \mathrm{W}\right)=\mathrm{Rsi}+\mathrm{R} 1+\mathrm{R} 2+\ldots+$ $+\mathrm{Rn}+$ Rse

Donde Rsi y Rse son las resistencias térmicas superficiales correspondientes al aire 
interior y exterior respectivamente y R1, R2 y $\mathrm{Rn}$ son las resistencias térmicas de cada una de las capas interiores del cerramiento. Las resistencias térmicas de las diferentes capas homogéneas que configuran las soluciones constructivas sobre las que realizamos los cálculos están extraídas del Catálogo de Elementos Constructivos del Código Técnico (33), siendo necesario tan sólo el cálculo de la resistencia térmica de la capa de aislamiento en función de la conductividad térmica del aislante empleado y del espesor del mismo.

Por su parte, la resistencia térmica de una capa térmicamente homogénea, como es el caso de la capa de aislamiento, se calcula a partir de la siguiente expresión:

$\mathrm{R}=e / \lambda$

Donde e es el espesor de la capa (m) y $\lambda$ es la conductividad térmica de diseño del material $(\mathrm{W} / \mathrm{mK})$ calculada a partir de valores térmicos declarados según norma UNE-EN ISO 10456:2001 (34). Materiales y productos para la edificación. Procedimientos para la determinación de los valores térmicos declarados y de diseño.

\subsubsection{Cálculo de la transmitancia térmica de las tres soluciones constructivas tipo}

En primer lugar, se determina el espesor del aislamiento térmico según la zonificación climática en la que se ubica el cerramiento.

Zona $\mathrm{B}=$ espesor $\mathrm{e}=0,035 \mathrm{~m}$

A continuación se calcula su conductividad térmica de diseño, $\lambda$, a partir del valor declarado del producto $\lambda^{\prime}$ según la norma UNE-EN ISO 10456:2001 (34).

- Valor declarado de la lana mineral: $\lambda^{\prime}=0,035 \mathrm{~W} / \mathrm{mK}$

- Coeficiente de conversión de humedad para lana mineral:

$\mathrm{F} \psi=4,00 \mathrm{~m}^{3} / \mathrm{m}^{3} ; \psi=0,02 \mathrm{~m}^{3} / \mathrm{m}^{3}$

Se ha supuesto un contenido de humedad por unidad de volumen $\psi=0,02 \mathrm{~m}^{3} / \mathrm{m}^{3}$, dentro de la limitación $\psi<0,15 \mathrm{~m}^{3} / \mathrm{m}^{3}$ establecido para la lana mineral (34).
- Factor de conversión (Fm):

$F m=e^{F \psi(\psi 2+\psi 1)} ; F m=e^{4,00(0,02-0)}=1,083$

- Conductividad térmica convertida a valor de diseño:

$\lambda=0,038 \mathrm{~W} / \mathrm{mK}$

Obtenidos el espesor y la conductividad térmica del aislamiento empleado en la solución constructiva, se procede al cálculo de su resistencia térmica como capa homogénea del conjunto constructivo (RAT), para posteriormente incorporarla a la resistencia térmica total proporcionada por el Catálogo de Elementos Constructivos (RT).

RAT $=\mathrm{e} / \lambda ;$ RAT $=0,921 \mathrm{~m}^{2} \mathrm{~K} / \mathrm{W}$

Finalmente, la transmitancia térmica de la solución constructiva se calcula a partir de la siguiente expresión definida también en el catálogo (33):

$U=1 /(0,710+R A T) ; U=0,613 \mathrm{~W} / \mathrm{m}^{2} \mathrm{~K}$

Una vez realizados los cálculos, es necesario comprobar que el valor obtenido para este cerramiento se encuentra dentro del valor límite de transmitancia térmica de muros de fachada marcado para la zona climática B4 objeto de estudio: $\mathrm{UMlim}=0,820 \mathrm{~W} / \mathrm{m}^{2} \mathrm{~K}$.

De este modo, queda justificado que la solución constructiva tipo uno cumple con la limitación de demanda energética establecida por el CTE.

Aplicando la misma metodología antes descrita, se comprueba que las tres soluciones constructivas analizadas cumplen con las exigencias establecidas por el CTE, tal y como se recoge en la Tabla 4.

\subsection{Desarrollo de las soluciones constructivas de fachada trasdosada}

Realizado el estudio de identificación y cálculo de la transmitancia térmica de las tres soluciones de fachada anteriormente expuestas se concluye que la que presenta las características más propicias para emplear en la fabricación del prototipo es la que muestra su hoja interior de yeso laminado, pues esta solución, además de presentar un valor aceptable de transmitancia

Tabla 4

Transmitancia térmica de las fachadas objeto de estudio

\begin{tabular}{|l|c|c|c|c|c|}
\hline & \multicolumn{5}{|c|}{ Características térmicas de los cerramientos andaluces tipo } \\
\cline { 2 - 6 } & $\begin{array}{c}\text { Resistencia térmica } \\
\text { de los restantes materiales } \\
\mathrm{R}\left(\mathrm{m}^{2} \mathrm{~K} / \mathrm{W}\right)\end{array}$ & $\begin{array}{c}\text { Resistencia térmica } \\
\text { del aislamiento } \\
\mathrm{R}_{\mathrm{TA}}\left(\mathrm{m}^{2} \mathrm{~K} / \mathrm{W}\right)\end{array}$ & $\begin{array}{c}\text { Resistencia térmica total } \\
\mathrm{R}_{\mathrm{T}}\left(\mathrm{m}^{2} \mathrm{~K} / \mathrm{W}\right)\end{array}$ & $\begin{array}{c}\text { Transmitancia térmica } \\
\mathrm{U}\left(\mathrm{W} / \mathrm{m}^{2} \mathrm{~K}\right)\end{array}$ & $\begin{array}{c}\text { Transmitancia térmica máxima } \\
\text { de cerramientos en zona } \\
\mathrm{B} 4 \mathrm{U} \leq 0,82 \mathrm{~W} / \mathrm{m}^{2} \mathrm{~K}\end{array}$ \\
\hline Solución 1 & 0,710 & 0,921 & 1,631 & 0,613 & 0,671 \\
\hline Solución 2 & 0,570 & 0,921 & 1,149 & 0,684 & $\mathrm{CUMPLE}$ \\
\hline Solución 3 & 0,540 & 0,921 & 1,461 & $\mathrm{CUMPLE}$ \\
\hline
\end{tabular}


5. Perspectiva de la solución constructiva. térmica $\left(\mathrm{U}=0,671 \mathrm{~W} / \mathrm{m}^{2} \mathrm{~K}\right)$, se encuentra ejecutada con paneles de yeso de características formales y dimensionales similares a los paneles desarrollados con los nuevos materiales reciclados que se estudian en el presente trabajo.

\subsubsection{Descripción de las soluciones constructivas}

El prototipo se encuentra compuesto por una hoja principal exterior de fábrica de ladrillo cerámico hueco de espesor 11,50 $\mathrm{cm}$, revestido por el exterior con un enfoscado de mortero continuo de $1,50 \mathrm{~cm}$ de espesor, cámara de aire no ventilada de $3,00 \mathrm{~cm}$ de espesor, aislamiento térmico de lana mineral adosado a la hoja interior del cerramiento de 3,50 cm de espesor y una hoja interior autoportante constituida a partir de los paneles de 1,50 $\mathrm{cm}$ de espesor realizadas con los nuevos materiales reciclados compuestos fundamentalmente por cenizas procedentes de la combustión de orujillo y del carbón en centrales térmicas, respectivamente.

Debido a la necesidad técnica de mantener la separación física de tres centímetros entre las hojas exterior e interior del cerramiento que configura la cámara de aire no ventilada, se ha de emplear una estructura metálica autoportante que sirva de base de sustentación de los paneles que conforman la hoja interior (35). Dicha estructura autoportante está compuesta por perfiles metálicos de dos tipologías diferentes:

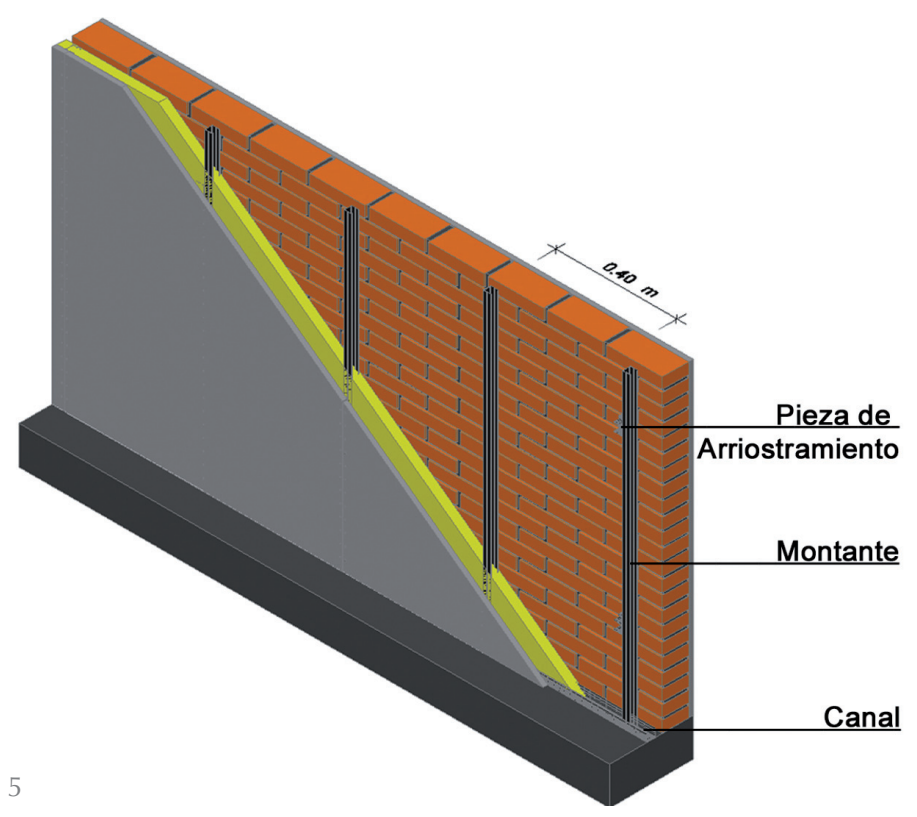

- Los canales: son los elementos de anclaje a suelo y techo de los perfiles verticales (o montantes) que soportan los paneles. Su anclaje sobre el soporte (suelo/techo) se realiza mediante tornillería; dichos tor- nillos se sitúan como máximo a $60 \mathrm{~cm}$ y la distancia del primer tornillo al extremo del canal es como máximo de $5 \mathrm{~cm}$. Bajo el canal inferior se coloca una junta o banda estanca que aísla del paso de las vibraciones y protege el canal de diversas agresiones, confiriéndole más estanqueidad y aminorando posibles puentes acústicos por desajuste en las uniones.

- Los montantes: estos perfiles se sitúan encajados en los canales del suelo y techo mediante un giro y sin atornillar, excepto los Ilamados montantes fijos situados en los arranques y encuentros, los cuales se atornillan al canal mediante tornillo MM (Metal-Metal). Se colocan a una distancia entre ejes de $40 \mathrm{~cm}$ y con una longitud 10 $\mathrm{mm}$ menor que la altura entre suelo y techo; las perforaciones en su alma, distanciadas $50 \mathrm{~cm}$ entre sí, están ideadas para permitir el paso de instalaciones.

\section{RESULTADOS Y DISCUSIÓN}

\subsection{Realización de los prototipos}

Una vez estudiada la solución constructiva para la sujeción de los paneles se procede al montaje de la misma con el fin de estudiar el comportamiento de los nuevos materiales en su puesta en obra, pudiendo obtener de esta manera datos sobre su aplicación técnica.

En primer lugar se realiza un pequeño forjado de hormigón a modo de solera que sirve como soporte de la solución constructiva, sobre el que se ejecuta el cerramiento exterior de fábrica de ladrillo cerámico hueco. La hoja exterior del prototipo se ha realizado con ladrillo hueco, a pesar de que en la solución de fachada del Catálogo de Elementos Constructivos del CTE seleccionada para aplicar la fórmula simplificada dicha hoja exterior se encuentra ejecutada en ladrillo perforado. Esto se debe a que la tipología de ladrillo del cerramiento exterior es irrelevante para cumplir con el objetivo que se pretende a la hora de realizar el prototipo, que no es otro que la comparación del comportamiento mecánico, durante la puesta en obra, de las dos placas objeto de estudio al atornillarlas al perfil metálico. Fraguado el cerramiento de fábrica se procede a disponer la estructura metálica portante que sirve de soporte a los paneles que constituyen la hoja interior del cerramiento.

Primero se realizan las perforaciones necesarias en el forjado para la sujeción del canal. Entre el forjado y el canal se coloca una banda de neopreno estanca que permite asegurar el ajuste en la unión entre los elementos, confiriendo protección al canal. 
Sujeto el canal se encaja el montante sobre el que se sujetan los paneles que configuran la hoja interior.

Por tratarse de una estructura autoportante además de los perfiles en la estructura metálica, se hace necesaria la colocación de alguna pieza para arriostrar dicha estructura al cerramiento de fábrica cerámica exterior. La pieza arriostrante se realiza disponiendo dos trozos de canal de $6,5 \mathrm{~cm}$ cada uno en ángulo recto y atornillando ambas piezas por sus laterales quedando sujetas en forma de escuadra. A continuación, esta pieza se coloca atornillada al montante y al cerramiento cerámico, de forma que arriostra la estructura metálica proporcionándole estabilidad.

Ejecutadas la hoja exterior del cerramiento y la estructura metálica portante que sostiene los paneles, se procede a colocar el material aislante, lana mineral, entre la perfilería de la estructura, de tal manera que quede contiguo a la hoja interior del cerramiento, evitando el contacto con el cerramiento exterior, con el fin de evitar que el material se humedezca, pues perdería sus propiedades aislantes. A continuación, se atornillan los paneles a la perfilería.
En la puesta en obra del material, en el caso de los paneles realizados con cenizas de orujillo, el corte de los mismos se realiza de forma rápida y sencilla con una simple sierra y su atornillado se realiza directamente al perfil con relativa facilidad. No obstante, en el caso de los paneles realizados con cenizas de carbón, surgieron algunos problemas durante su puesta en obra, pues tal como se aprecia en la Tabla 2 , donde se comparan los valores de sus resistencias mecánicas, el panel de cenizas de carbón muestra unos valores ligeramente inferiores a los del panel de cenizas de orujillo. Esta disminución de valores de resistencia mecánica se traduce durante su manipulación en una mayor rotura de piezas durante el corte de los paneles y durante su atornillado al montante de sujeción debido a la escasa distancia entre la arista exterior del panel y la línea de atornillado, que en el caso del prototipo fabricado con paneles de cenizas de orujillo. Así, la colocación de los paneles de cenizas de orujillo se realiza satisfactoriamente, mientras que la de los paneles de cenizas de carbón presenta problemas de fisuras que hacen necesaria la sustitución de los mismos en varias ocasiones.

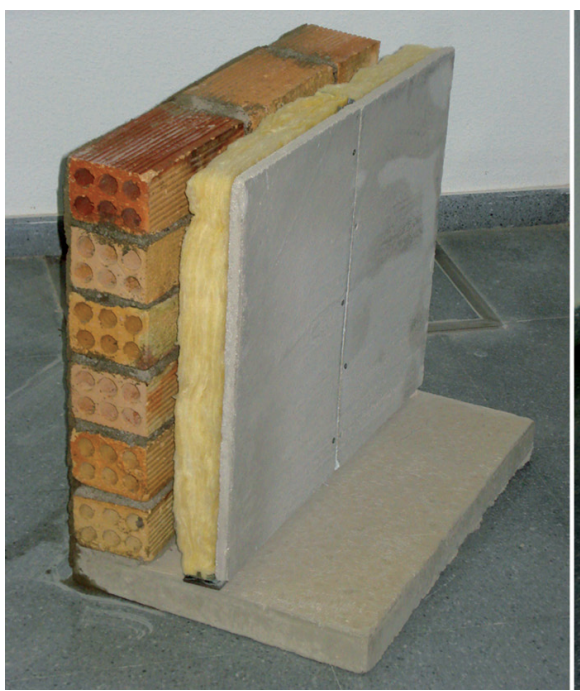

En el prototipo se ha realizado la unión de dos paneles en un mismo montante por tratarse de la unión más desfavorable, ya que las distancias entre la línea de atornillado y el borde lateral del panel es mínima. De este modo se ha conocido la dureza y resistencia límite de atornillado de los paneles.

Para concluir el prototipo se realiza e revestimiento exterior. Esta capa se encuentra realizada sobre la parte exterior del cerramiento de fábrica de ladrillo, mediante un enfoscado continuo de mortero capaz de proporcionarle estanqueidad a la fábrica.

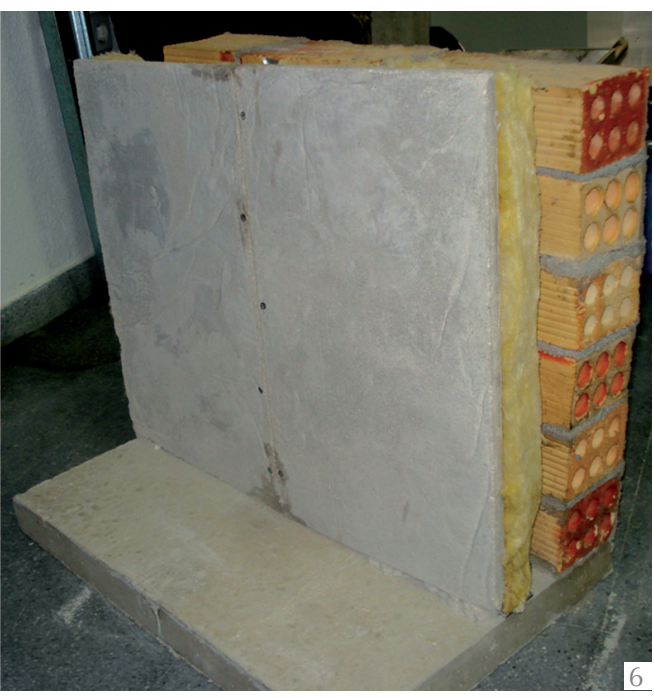

\subsection{Evaluación de la disposición de los paneles en la solución constructiva}

El cálculo de la transmitancia térmica de estas soluciones constructivas se realiza a partir de la misma formulación utilizada para el cálculo de la transmitancia térmica de la solución constructiva tipo dos cuya hoja interior se encuentra realizada con paneles de yeso laminado, dadas las muchas similitudes formales, constructivas y técnicas de dicho trasdosado con respecto a los nuevos paneles de material reciclado.

$U=1 /\left(0,570+R_{A T}\right)$
6. Prototipo de la solución constructiva de fachada con los paneles de cenizas de orujillo (izq.) y paneles de ceniza de carbón (dcha.). 
A partir de esta fórmula simplificada se deducen las correspondientes a los nuevos paneles de cenizas de orujillo y de cenizas de carbón. Para ello, basta sustraerle al valor $0,570 \mathrm{~m}^{2} \mathrm{~K} / \mathrm{W}$, correspondiente a la suma de las resistencias térmicas de los diferentes materiales que componen la solución, la resistencia térmica correspondiente a los paneles de yeso R paneles de yeso $=0,083 \mathrm{~m}^{2} \mathrm{~K} / \mathrm{W}$ y sumarle la resistencia térmica de los paneles de cenizas de orujillo, $\mathrm{R}$ paneles de cenizas de orujillo $=0,058 \mathrm{~m}^{2} \mathrm{~K} / \mathrm{W}$, y de los paneles de cenizas de carbón, $R$ paneles de cenizas de carbón=0,054 $\mathrm{m}^{2} \mathrm{~K} / \mathrm{W}$, respectivamente. De este modo, se obtienen las siguientes fórmulas para el cálculo de la transmitancia térmica de las nuevas soluciones constructivas propuestas:

$U$ paneles de cenizas de orujillo $=1 /(0,543+$ RAT $)$

$U$ paneles de cenizas de carbón $=1 /(0,540+$ RAT $)$

A continuación se lleva a cabo el cálculo de las transmitancias térmicas de las nuevas soluciones constructivas mediante el procedimiento expuesto en el apartado 3.1, a partir del que se obtiene un valor de resistencia térmica de la capa de aislamiento térmico (RAT) de 0,921 m²K/W considerando la zonificación climática B. Las transmitancias térmicas de las nuevas soluciones constructivas realizadas con los paneles de material reciclado se obtienen sustituyendo este dato en las expresiones anteriores:

$\mathrm{U}$ paneles de cenizas de orujillo $=0,683 \mathrm{~W} / \mathrm{m}^{2} \mathrm{~K}$

$\mathrm{U}$ paneles de cenizas de carbón $=0,684 \mathrm{~W} / \mathrm{m}^{2} \mathrm{~K}$

Finalmente, se aprecia que el comportamiento térmico de las nuevas soluciones constructivas elaboradas a partir de paneles de materiales reciclados es ligeramente inferior al de la solución realizada con paneles de yeso $\left(\mathrm{U}=0,671 \mathrm{~W} / \mathrm{m}^{2} \mathrm{~K}\right)$ al ser sus transmitancias térmicas superiores; no obstante, cabe resaltar que dichas transmitancias térmicas se encuentran dentro de los límites establecidos por el CTE al no rebasar los 0,820 W/m² K fijados para la zona climática de Sevilla.

\section{CONCLUSIONES}

Los resultados obtenidos en el presente trabajo permiten establecer las siguientes conclusiones:

- La utilización de los materiales desarroIlados no representa ningún riesgo para el medio ambiente, pues se ha determinado a través de ensayos de lixiviación que a pesar del elevado contenido de material residual su impacto es mínimo.

- Comparando los valores de las propiedades mecánicas de los paneles objeto de estudio en el presente trabajo, se concluye que los paneles de cenizas de orujillo en general muestran unos valores ligeramente superiores de dichas propiedades mecánicas que los paneles de cenizas de carbón, lo que se traduce en un mejor comportamiento durante el proceso de puesta en obra, tal como se demuestra tras la realización de los prototipos. La resistencia a flexión y compresión de los paneles de cenizas de orujillo permite su perforación y atornillado incluso en las zonas más desfavorables sin que se produzcan roturas en los mismos.

- La transmitancia térmica de las nuevas soluciones constructivas realizadas son ligeramente superiores a la solución realizada con paneles de yeso. No obstante, ambos valores se encuentran dentro de los límites establecidos en el CTE para la zona climática B4 objeto de estudio por lo que cumplen con las limitaciones de demanda energética dispuestas en la normativa vigente.

- Los valores de transmitancia térmica obtenidos para las tres soluciones de fachada estudiadas quedan próximos a los límites establecidos en el CTE para la zona climática B4, por lo que ante la posible existencia de puentes térmicos podrían sobrepasar fácilmente este valor límite, incumpliendo así lo establecido en el CTE. Esto se solucionaría incorporando mayores espesores en los aislamientos que permitan una holgura mayor respecto al valor límite de la zona objeto de estudio.

- La implantación de estos paneles en una solución plenamente integrada en el sistema constructivo de cerramiento, los convierte en un nuevo material técnicamente viable y económicamente competitivo dentro del mercado de los materiales de construcción, al suponer su mayoritaria composición a base de residuos un importante ahorro en la extracción de materia prima.

- El empleo de residuos procedentes de la combustión en centrales térmicas de orujillo y de carbón en nuevos materiales de construcción, como los paneles para trasdosados interiores de cerramientos presentados, supone un gran avance hacia la construcción sostenible ya que se consigue, al mismo tiempo, minimizar la explotación de los recursos naturales y fomentar el empleo de materiales recicla- 
dos en la fabricación de nuevos productos para el sector de la edificación.

En trabajos futuros se propone la realización de un nuevo prototipo a escala real, con los paneles ejecutados con cenizas de orujillo, que permita estudiar la eficacia de este nuevo material como solución de trasdosado y partición interior, en forma de paneles de cartón-yeso, ensayando la permeabilidad del aire, la resistencia al fuego y la transmitancia del conjunto. De este modo se conseguiría cotejar si es posible la fabricación y montaje de los paneles a escala real y la viabilidad de introducir el nuevo material en el mercado de los pro- ductos de construcción, cuyo principal valor añadido estriba en los beneficios ambientales que incorpora mediante la reducción del consumo de recursos naturales y la eliminación de residuos procedentes de centrales térmicas, logrando así un menor impacto sobre el territorio de los sectores de la edificación y la industria.

\section{AGRADECIMIENTOS}

Esta investigación se ha realizado dentro del proyecto ARCEVA, subvencionado por la Junta de Andalucía, Consejería de Vivienda y Ordenación del Territorio (Boja n. ${ }^{\circ} 94$ del 13 de mayo de 2008).

\section{BIBLIOGRAFÍA}

(1) Rodríguez-Saumell, J.: Tipología de muros, fachadas y valores de significación en la arquitectura. Universidad de Sevilla, Secretariado de Publicaciones, Instituto Universitario de Ciencias de la Construcción, Sevilla, 1998.

(2) Garí, J. y Soto, S.: "Cerramientos verticales-fachadas", p.197, Ceac, Barcelona, 2005.

(3) Gómez Jáuregui, V.: "Fachadas con mucha historia". Gremios. Periódico de la Construcción, vol. 73 (2008), pp. 32-33.

(4) Ros-García, J. M.: "La fábrica de doble hoja en Madrid, un siglo de cerramiento moderno". Informes de la Construcción, Vol. 56 n. ${ }^{\circ} 495$ (2005), pp. 57-71. Doi: 10.3989/ ic.09.000 ER.

(5) Villanueva de, L.: "Evolución histórica de la construcción con yeso". Informes de la Construcción, vol. 56, n. ${ }^{\circ} 493$ (2004), pp. 5-11. Doi: 10.3989/ic.09.000 ER.

(6) Código Técnico de la Edificación (CTE), p.1255, Ministerio de Vivienda, Madrid, 2009.

(7) Pérez Bernal, I.: Eco productos: en la arquitectura y el diseño, p. 252, AxE-Arquitectura y Entorno, S.L., Barcelona, 2008.

(8) EN 450-1:2005: Ceniza para hormigones. Parte 1: Definiciones, especificaciones y criterios de conformidad. Comité Europeo de Normalización (CEN), Bruselas, 2005.

(9) Radian Canada, Inc. and CANMET: Investigation of high-volume fly ash concrete systems. EPRI Report n. ${ }^{\circ}$ TR-10315, 1993.

(10) European Coal Combustion Products Association. Disponible en: http://www.ecoba. com/[2010, 6/5/2010].

(11) Leiva, C.: Tesis doctoral: Desarrollo y especificaciones técnicas de productos ignífugos fabricados a partir de residuos industriales, para su uso como elementos constructivos de separación. Universidad de Sevilla, Sevilla, 2006.

(12) Leiva, C.; Vilches, L. F.; Vale J. y Fernández-Pereira, C.: "Influence of the type of ash on the fire resistance characteristics of ash-enriched mortars". Fuel, vol. 84 n. ${ }^{0} 11$ (2005), pp. 1433-1439. doi:10.1016/j.fuel.2004.08.031.

(13) Vilches, L. F.; Leiva, C.; Vale, J.; Fernández-Pereira, C.: "Insulating capacity of fly ash pastes used for passive protection against fire". Cement and Concrete Composites, vol. 27, n. ${ }^{\circ}$ 7-8 (2005), pp. 776-781. doi:10.1016/cemconcomp.2005.03.001.

(14) Leiva, C.; Vale, J.; Vilches, L. F.; Fernández Pereira, C y Olivares del Valle, J.: Obtención de materiales aislantes a partir de residuos de procesos térmicos que utilizan biomasa. Oficina Española de Patentes y Marcas, n. ${ }^{\circ}$ Solicitud: P200401702, n. ${ }^{\circ}$ Publicación: 2245895. Disponible en: http://invenes.oepm.es/InvenesWeb/detalle?referencia= p200401702 [2010, 6/5/2010].

(15) Miranda, L. F. R.; Selmo, S. M. S.: "CDW recycled aggregate renderings: Part I - Analysis of the effect of materials finer than $75 \mu \mathrm{m}$ on mortar properties". Construction and Building Materials, vol. 20, n. ${ }^{\circ} 9$ (2006), pp. 615-624. doi:10.1016/j.conbuildmat.2005.02.025.

(16) Meyer, C.: "The greening of the concrete industry". Cement \& Concrete Composites, vol. 31, n. 8 (2009), pp.601-605. doi:10.1016/j.cemconcomp.2008.12.010.

(17) Berndt, M. L.: "Properties of sustainable concrete containing fly ash, slag and recycled concrete aggregate". Construction and Building Materials, vol. 23, n. 7 (2009), pp. 2606-2613. doi: 10.1016/j.conbuildmat.2009.02.011.

(18) González-Madariaga, F. J. y Lloveras-Macia, J.: "Mezclas de residuos de poliestireno expandido (EPS) conglomerados con yeso o escayola para su uso en la construcción". Informes de la Construcción, vol. 60, n. ${ }^{\circ} 509$ (2008), pp. 35-43. 
(19) Río-Merino del, M. y Domínguez, J.: "Escayola aligerada con sólidos celulares". Informes de la Construcción, vol. 50, n. ${ }^{\circ} 458$ (1998), pp. 43-60. Doi:10.3989/ic.09.000 ER.

(20) Río-Merino del, M.; Hernández-Olivares, F. y Comino-Almenara, P.: "Estado del arte sobre el comportamiento físico-mecánico de la escayola reforzada con fibras de vidrio $E^{\prime \prime}$. Informes de la Construcción, vol. 56, n. 493 (2004), pp. 33-37. Doi:10.3989/ic.09.000 ER.

(21) García Santos, A.: "Caracterización de compuestos de escayola reforzados, en relación con el tipo de refuerzo y la relación A/Y". Informes de la Construcción, vol. 56, n. ${ }^{\circ} 493$ (2004), pp. 19-31. Doi:10.3989/ic.09.000 ER.

(22) UNE 102001:1986. Aljez o piedra de yeso. Clasificación. Características. Asociación Española de Normalización y Certificación (AENOR), Madrid, 1986.

(23) UNE-EN 12859:2009. Paneles de yeso: Definiciones, especificaciones y métodos de ensayo. Asociación Española de Normalización y Certificación (AENOR), Madrid, 2009.

(24) ASTM E761 - 92(2005). Standard test method for compressive strength of sprayed fireresistive material applied to structural members. American Society for Testing and Materials (ASTM), Pensilvania, 2005.

(25) ASTM C348 - 08. Standard test method for flexural strength of hydraulic-cement mortars. American Society for Testing and Materials (ASTM), Pensilvania, 2008.

(26) UNE-EN 993-15:2005. Métodos de ensayo para productos refractarios conformados densos. Parte 15: Determinación de la conductividad térmica mediante el método del hilo caliente (paralelo). Asociación Española de Normalización y Certificación (AENOR), Madrid, 2005.

(27) UNE-EN 1363-1:2000. Ensayos de resistencia al fuego. Parte 1: Requisitos generales. Asociación Española de Normalización y Certificación (AENOR), Madrid, 2000.

(28) Vilches, L. F.; Leiva, C.; Vale, J.; Olivares, J. y Fernández-Pereira, C.: "Fire resistance characteristics of plates containing a high biomass-ash proportion". Industrial \& Engineering Chemistry Research, vol. 46, n. ${ }^{\circ} 14$ (2007), pp. 4824-4829. Doi:10.1021/ie061194f.

(29) Soil Quality Decree. Decree n. 469 of 2007 containing rules relative to quality of soil. Besluit van 22 november 2007, houdende regels inzake de kwaliteit van de bodem (Besluit bodemkwaliteit). p. 137, Staatsblad van het Koninkrijk der Nederlanden, La Haya, 2007.

(30) NEN 7345: Leaching characteristics of soil an stony building and waste materials-Leaching test-Determination of the leaching of inorganic components from building and monolithic waste materials with the diffusion test. Netherlands Standardization Institute, Delft, 1995.

(31) Mercader, M. P.; Marrero, M.; Solís, J.; Montes, M. V. y Ramirez, A.: "Cuantificación de los recursos materiales consumidos en la ejecución de la cimentación". Informes de la Construcción, vol. 62, n. 517 (2010), pp.125-132. Doi:10.3989/ic.09.000 ER.

(32) Mercader Moyano, M. P.: Tesis doctoral: Cuantificación de los recursos consumidos y emisiones de CO2 producidas en las construcciones de Andalucía y sus implicaciones en el Protocolo de Kyoto. Universidad de Sevilla, Sevilla, 2010.

(33) Catálogo de Elementos Constructivos del Código Técnico de la Edificación v1.2. Ministerio de Vivienda, Instituto de Ciencias de la Construcción Eduardo Torroja, CSIC, Madrid, 2010. Disponible en: http://www.elementosconstructivos.codigotecnico.org/ [2010, 6/5/2010].

(34) UNE-EN ISO 10456:2001. Materiales y productos para la edificación. Procedimientos para la determinación de los valores térmicos declarados y de diseño. (ISO 10456:1999). Asociación Española de Normalización y Certificación (AENOR), Madrid, 2001.

(35) Catálogo de soluciones acústicas y térmicas para la edificación. Elementos constructivos con placas de yeso laminado y lana mineral. ATEDY y AFELMA, Madrid, 2009. 